\title{
Looks aren't everything, but neither is microRNA profiling
}

For patients with head and neck squamous cell carcinoma (HNSCC), metastasis occurs synchronously in $2 \%$ to $17 \%$ and metachronously in $10 \%$ to $40 \% ; 70 \%$ to $85 \%$ of the metastases are in the lungs. ${ }^{1}$ With similar risk factors and demographics, difficulty in histologic differentiation between primary lung squamous cell cancer (LSCC) and HNSCC metastasis has driven interest in molecular profiling. ${ }^{2} \mathrm{Mu}-$ noz-Largacha and colleagues ${ }^{3}$ have asked a clinical relevant question by querying whether a micro (mi)RNA profile can differentiate HNSCC from primary LSCC.

miRNA profiling is a molecular technology under evaluation for biomarkers in multiple cancers. ${ }^{4}$ Regulatory RNA sequences act in both oncogenic and tumor suppressive manners by binding mRNA transcripts and regulating protein expression; they are detectable via liquid biopsy and tumor samples. ${ }^{5,6}$ Examples exist in LSCC, including the report by Chen and colleagues ${ }^{7}$ demonstrating that miR-375 is a tumor suppressor, and the report by Gao and colleagues ${ }^{8}$ identifying a 7-miRNA signature associated with overall survival. Muñoz-Largacha and colleagues $^{3}$ reported that 3 miRNAs and the ratio of 2 (miR-10a and 10b) differentiated LSCCs from HNSCC. Their analysis benefitted from internal calibration based on the ratio of miR-10a and $10 \mathrm{~b}$, which eliminated housekeeping gene normalization. In addition, they reported a correlation to The Cancer Genome Atlas. However, significant limitations exist.

The comparison in this article ${ }^{3}$ is not between primary LSCC and metastatic HNSCC, rather between primary LSCC and primary HNSCC. The diagnostic dilemma is differentiating metastatic HNSCC with primary LSCC, not differentiating primary tumors. miRNA profiles are associated with the epithelial to mesenchymal transition and metastatic potential; they will be different between primary lesions and metastatic disease from the same tumor. ${ }^{9}$ Muñoz-Largacha and colleagues ${ }^{3}$ state this limitation. They report that "myo-miRs" associated with striated muscle were found in the primary HNSCC samples versus miRNA associated with multiciliated cells in LSCC samples. These findings suggest that the miRNA profile of future.

\section{References} 86:1-9.

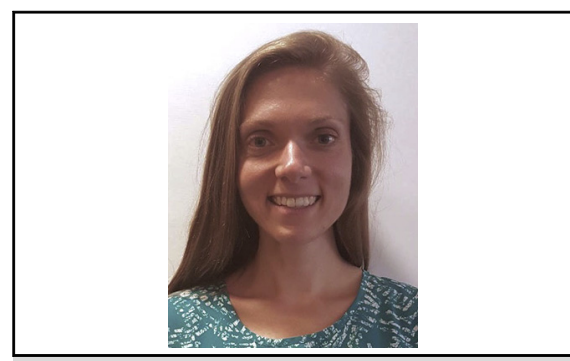

Kaitlin C. McLoughlin, MD

\section{Central Message}

miRNA profiling may be a beneficial tool in the differentiation of metastatic head and neck from primary LSCC, but not yet.

See Article page 714

an HNSCC metastasis is unlikely to be synonymous with primary HNSCC. The tumor microenvironment is a limitation of miRNA biomarker discover. Clearly, the environment is different between the neck and lungs. Ideally, laser microdissection would be performed before miRNA analysis to overcome microenvironmental contamination of the specimens, but analysis between HNSCC lung metastasis and primary LSCC is more achievable. The reproducibility of miRNA is another limitation of this study and the field of miRNA discovery. miRNA profiling of HNSCC has been performed with as many associated miRNA signatures as studies. The heterogeneity is compounded by the differences in diagnoses from the oral cavity to the larynx, which is demonstrated with the differences of miR-10a and miR-34a based on anatomic site. ${ }^{10}$

These limitations prevent this technology or the specifically identified miRNAs in this article from being applied to management decisions for patients. However, the attempt to tackle this field by Muñoz-Largacha and colleagues $^{3}$ is a worthwhile research endeavor that should be continued and hopefully will benefit patients in the

1. Takes RP, Rinaldo A, Silver CE, Haigentz M Jr, Woolgar JA, Triantafyllou A, et al. Distant metastases from head and neck squamous cell carcinoma. Part I. Basic aspects. Oral Oncol. 2012;48:775-9.

2. Mercer RR, Lucas NC, Simmons AN, Zander DS, Tsongaliz GJ, Funkhouser WK, et al. Molecular discrimination of multiple primary versus metastatic squamous cell cancers of the head/neck and lung. Exp Mol Pathol. 2009;

3. Muños-Laracha JA, Gower AC, Sridhar P, Deshpande A, O'Hara CJ, Yamada E, et al. miRNA profiling of primary lung and head and neck squamous cell 
carcinomas: addressing a diagnostic dilemma. J Thorac Cardiovasc Surg. 2017; 154:714-27.

4. Mishra S, Yadav T, Rani V. Exploring miRNA based approaches in cancer diagnostics and therapeutics. Crit Rev Oncol Hematol. 2016;98:12-23.

5. Gao X, Wang Y, Zhao H, Wei F, Zhang X, Su Y, et al. Plasma miR-324-3p and miR-1285 as diagnostic and prognostic biomarkers for early stage lung squamous cell carcinoma. Oncotarget. 2016;7:59664-75.

6. Chen X, Ba Y, Ma L, Cai X, Yin Y, Wang K, et al. Characterization of microRNAs in serum: a novel class of biomarkers for diagnosis of cancer and other diseases. Cell Res. 2008;18:997-1006.

7. Chen WJ, Gan TQ, Qin H, Huang SN, Yang LH, Fang YY, et al. Implication of downregulation and prospective pathway signaling of
microRNA-375 in lung squamous cell carcinoma. Pathol Res Pract 2017;213:364-72.

8. Gao X, Wu Y, Yu W, Li H. Identification of a seven-miRNA signature as prognostic biomarker for lung squamous cell carcinoma. Oncotarget. 2016;7: 81670-9.

9. Li JZ, Gao W, Lei WB, Zhao J, Chan JY, Wei WI, et al. MicroRNA 7444-3p promotes MMP-9-mediated metastasis by simultaneously suppressing PDCD4 and PTEN in laryngeal squamous cell carcinoma. Oncotarget. 2016; 7:58218-33

10. Koshizuka K, Hanazawa T, Fukumoto I, Kikkawa N, Okamoto Y, Seki N The microRNA signatures: aberrantly expressed microRNAs in head and neck squamous cell carcinoma. J Hum Genet. 2017;62:3-13. 